\title{
Síndrome de piel escaldada por estafilococo
}

\section{Staphylococcal scalded skin syndrome}

\author{
Pedro Rivero Borrell-de la Parra, ${ }^{*}$ José Alberto Carranco-Dueñas, ${ }^{\ddagger}$ \\ Rosa Edith Gálvez-Martínez, ${ }^{*}$ Mariano Rivera-Echegoyen ${ }^{\S}$
}

Citar como: Rivero BPP, Carranco-Dueñas JA, Gálvez-Martínez RE, Rivera-Echegoyen M. Síndrome de piel escaldada por estafilococo. An Med ABC. 2021; 66 (2): 146-150. https://dx.doi.org/10.35366/100486

\begin{abstract}
RESUMEN
El síndrome de la piel escaldada por estafilococos es una afección que ataca predominantemente a los niños y causa un espectro variado de deterioros cutáneos, es causado por cierta toxina exfoliativa producida por aproximadamente el $5 \%$ de S. aureus. Es una manifestación de exfoliación aguda de la piel, secundaria a una infección inicial. Se presenta el caso de un niño de tres años con este diagnóstico, el paciente respondió adecuadamente al tratamiento, el cual incluía un antibiótico (cefalosporina de primera generación), un analgésico (paracetamol) e hidratación con líquidos intravenosos. Fue dado de alta después de siete días, completando dos semanas totales de tratamiento antibiótico, con una resolución casi completa de sus deterioros en la piel. Es importante tener una alta sospecha clínica de síndrome de piel escaldada por estafilococos para tener un dictamen y atención tempranos, disminuyendo así la morbilidad de este padecimiento.

Palabras clave: Staphylococcus aureus, síndrome de la piel escaldada, infección de piel.
\end{abstract}

\footnotetext{
${ }^{*}$ Médico Especialista en Pediatría. Adscrito al Servicio de Pediatría.

‡ Médico Especialista en Infectología Pediátrica.

$\S$ Médico Especialista en Gastroenterología Pediátrica.
}

Centro Médico ABC campus Santa Fe. México.

Recibido: 18/12/2019. Aceptado: 10/02/2020.

Correspondencia: Pedro Rivero Borrell-de la Parra

E-mail: peribopa@gmail.com

Abreviaturas:

S. aureus = Staphylococcus aureus.

SSSS $=$ Síndrome de piel escaldada por estafilococo.

DG- = Desmogleína-1.

CA-MRSA = Staphylococcus aureus resistente a meticilina adquirida en la comunidad.

TEN = Necrólisis epidérmica tóxica.

SJS = Síndrome de Stevens Johnson .

\begin{abstract}
Staphylococcal scalded skin syndrome is a bacterial toxin mediated skin disorder that primarily affects young children. The current case report describes the clinical progress of a patient who presented this syndrome. Based on clinical and paraclinical exams the diagnosis was established. He responded well to antibiotic treatment with a first generation cephalosporin, acetaminophen and intravenous fluids with full resolution in two weeks. Hence, early diagnosis and prompt treatment are very important for a successful outcome and reduce the morbidity of this syndrome.
\end{abstract}

Keywords: Staphylococcus aureus, staphylococcal scalded skin syndrome, skin infection.

\section{INTRODUCCIÓN}

El Staphylococcus aureus (S. aureus) forma parte de la flora humana normal, siendo responsable de una gran variedad de enfermedades cuyo rango va desde contaminaciones menores de la piel y tejidos blandos hasta infecciones graves que ponen en peligro la vida, como bacteriemia, endocarditis, pericarditis, neumonía, empiema, osteomielitis, miositis y artritis séptica.

El síndrome de la piel escaldada por estafilococo (SSSS) es causado por una toxina exfoliativa producida por aproximadamente el $5 \%$ del citado germen. 
Es una manifestación de exfoliación aguda de la piel, secundaria a un contagio primigenio, la cual frecuentemente ocurre en la cavidad oral o nasal, la garganta o el ombligo, seguido de una celulitis eritematosa.

La gravedad de dicho síndrome varía, desde unas pocas ampollas localizadas en el sitio de la infección, hasta un desgaste grave que afecta a casi todo el cuerpo.

Los menores de cinco años de edad tienen un alto riesgo de padecer SSSS debido a que en la infancia es cuando se adquiere la inmunidad de por vida, en forma de anticuerpos, contra las exotoxinas. ${ }^{1-3}$

\section{CASO CLÍNICO}

Se trata de paciente masculino de tres años 11 meses, previamente sano, producto de embarazo gemelar resuelto sin complicaciones a las 36 semanas de gestación. Niega alergias.

Inicia su padecimiento actual dos semanas previas a su ingreso, con presencia de lesiones en narinas, tórax anterior y tórax posterior diagnosticadas como impétigo buloso, tratadas con amoxicilina con clavulanato, ácido fusídico, crema emoliente y solución de Burow, con lo cual presenta mejoría de daños en tórax, aunque no en las perinasales. Después de una semana de completar medicación antibiótica con amoxicilina y clavulanato, notan aumento de temperatura en piel de cuello, axilas, abdomen y genitales, evolucionando al día siguiente con incremento del eritema, acompañado de prurito. Acudió a consulta un día previo a su ingreso, en donde se

Figura 1:

Eritema auricular a nivel de la concha.

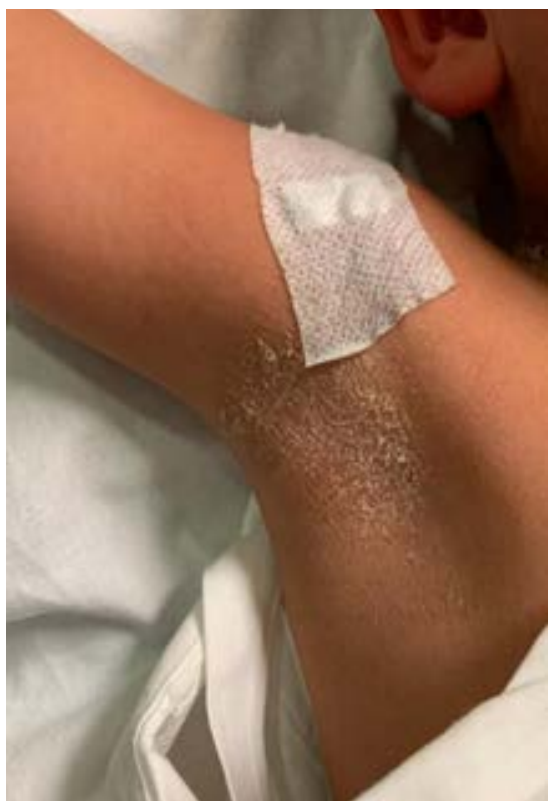

Figura 2:

Axila con placa eritematoescamosa

inició manejo con clindamicina vía oral y se aplicó hidrocortisona tópica, mejorando parcialmente la sintomatología cutánea. Al siguiente día acudió nuevamente a consulta y es referido con Dermatología Pediátrica, quienes sospechan síndrome de Stevens Johnson versus síndrome de piel escaldada estafilocócica por lo que es llevado al Servicio de Urgencias. Durante el padecimiento no hay historia de fiebre. Ingresó a hospitalización y se inició manejo por parte de dermatología con mupirocina periorificial dos veces al día, petrolato puro en zonas denudadas de piel y labios y también se realiza biopsia en brazo derecho por incisión con punch \#4. Se solicita interconsulta con oftalmología quienes indican ciprofloxacino una gota cada 8 horas en ambos ojos y hialuronato de sodio una gota tres veces por día en ambos ojos. Se gestiona interconsulta con infectología pediátrica, donde se sospecha de $S$. aureus meticilino resistente (MRSA) por la pobre respuesta a la amoxicilina con clavulanato y por la falta de mejoría clínica a pesar de tener leucocitos y reactantes de fase aguda normales, por lo que se sugiere iniciar doble esquema con cefalotina y vancomicina esperando mejoría clínica de lesiones cutáneas.

A su ingreso se observa dermatosis caracterizada por eritema con aumento de la temperatura local en cara, pabellones auriculares, cuello, espalda, tórax, brazos, antebrazos, genitales e ingles, de bordes mal definidos, con zona de escama fina en cara lateral derecha del cuello, así como placas eritematosas con costras meliséricas en narinas y surco subnasal (filtrum). 
Se observan labios xeróticos, fisurados y algunas costras hemáticas; máculas hipocrómicas en tórax anterior y posterior; palmas y plantas respetadas; hiperemia conjuntival leve, seca, con costra melisérica en canto del ojo izquierdo. Sin gingivitis, sin detrimentos en mucosa oral. Resto normal. (Figuras 1 a 4).

Las pruebas de laboratorio a su ingreso reportan biometría hemática con hemoglobina $13.8 \mathrm{~g} / \mathrm{dL}$, hematocrito $40.9 \%$, leucocitos 9,700 ; neutrófilos $64 \%$, linfocitos $28 \%$, monocitos $5 \%$, eosinófilos $2 \%$, plaquetas 472,000; proteína $\mathrm{C}$ reactiva $0.08 \mathrm{mg} / \mathrm{dL}$, procalcitonina $0.1 \mathrm{ng} / \mathrm{mL}$; velocidad de sedimentación globular $6 \mathrm{~mm} / \mathrm{h}$; glucosa $89 \mathrm{mg} / \mathrm{dL}$, nitrógeno ureico en sangre $10 \mathrm{mg} / \mathrm{dL}$, creatinina $0.5 \mathrm{mg} / \mathrm{dL}$, strep test negativo, exudado faríngeo con biota habitual.

Cultivo aeróbico de lesiones perinasales con desarrollo $S$. aureus meticilino sensible.

Cultivo de hisopado de narinas, axilas e ingles con desarrollo de dicha bacteria.

Hemocultivo periférico negativo.

Desde el primer día de administración de antibiótico presentó mejoría notable en las escoriaciones periorales y nasales. Se mantuvo afebril. A las 72 horas se reporta aislamiento de $S$. aureus meticilino sensible en cultivo aeróbico de erosiones perinasales y en cultivo de hisopado de narinas, axilas e ingles, dejando monoterapia con cefalexina. Se toman controles de laboratorio al sexto día de estancia reportando en biometría hemática hemoglobina $14.4 \mathrm{~g} / \mathrm{dL}$, hematocrito $43 \%$, leucocitos 12,400; neutrófilos $36 \%$, linfocitos $51 \%$, monocitos $7 \%$, eosinófilos $5 \%$, plaque-

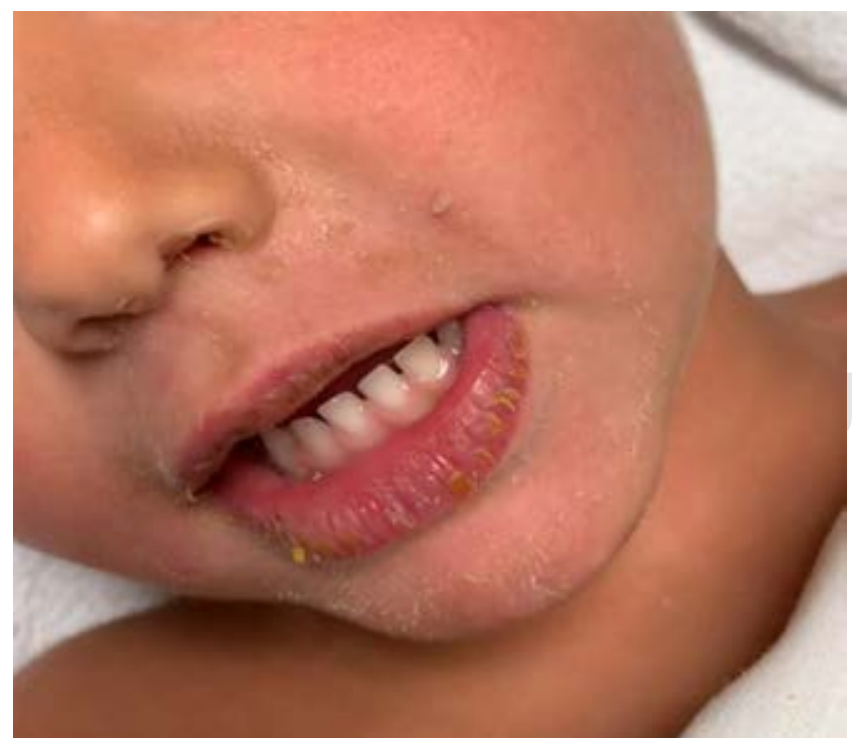

Figura 3: Descamación perioral.

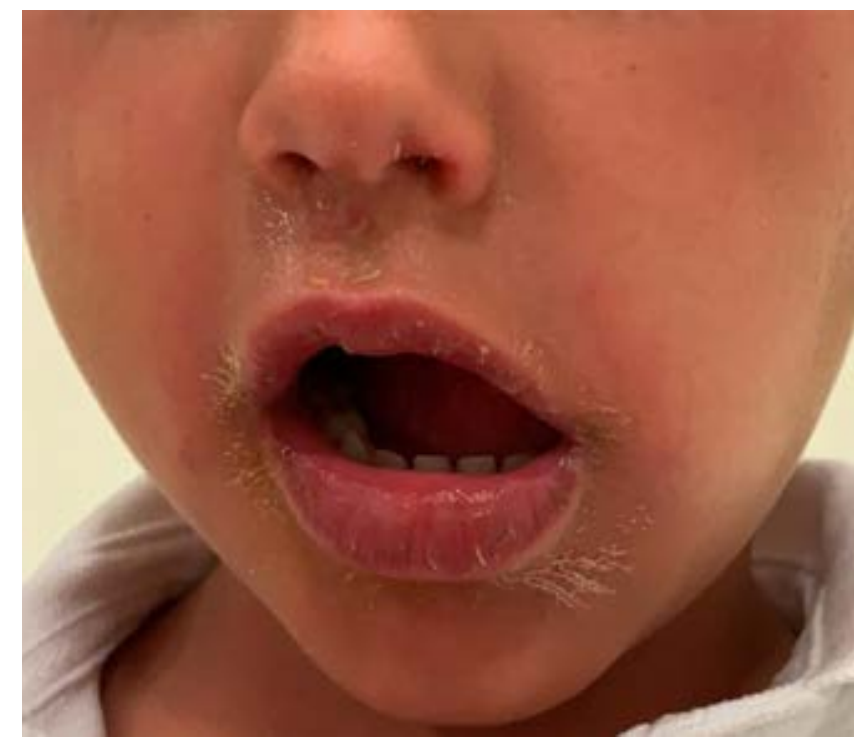

Figura 4: Descamación y fisuras periorales.

tas 467,000; proteína $\mathrm{C}$ reactiva $0.66 \mathrm{mg} / \mathrm{dL}$, procalcitonina $<0.05 \mathrm{ng} / \mathrm{mL}$, glucosa $89 \mathrm{mg} / \mathrm{dL}$, nitrógeno ureico en sangre $10 \mathrm{mg} / \mathrm{dL}$, creatinina $0.5 \mathrm{mg} / \mathrm{dL}$.

La biopsia reporta dermatitis espongiforme superficial por linfocitos con paraqueratosis y extravasación de eritrocitos.

Egresa al séptimo día de estancia con cefadroxilo $30 \mathrm{mg} / \mathrm{kg} /$ día en dos dosis, completando una semana más (en total 15 días). En consulta de seguimiento a la semana de egresado se observa sólo eritema en mejillas y pabellón auricular derecho con discreto aumento de la temperatura, sin edema de pabellón auricular. Hay escama fina en antihélix derecho, en rodillas, escama gruesa en dedos e interdigital bilateral. Sin hallazgos en fosas nasales.

\section{DISCUSIÓN}

El Staphylococcus aureus es un coco grampositivo que se presenta en pares, cadenas y racimos parecidos a aquellos de las uvas. Es ubicuo en la naturaleza y puede ser patógeno para humanos y animales. Son bacterias inmóviles, aeróbicas o anaeróbicas facultativas y se cultivan fácilmente en medios de laboratorio de rutina.

Las toxinas epidermolíticas producidas por especies de Staphylococcus actúan en un sitio remoto, lo cual lleva a una erupción eritematosa y la separación de la epidermis debajo de la capa de células granulares. Se forman bullas y se produce una descamación difusa, similar a una lámina. El SSSS es un conjunto 
de trastornos de exfoliación aguda de la piel secundario a una contaminación inicial que frecuentemente ocurre en cavidad oral o nasal, la garganta o el ombligo, seguido de una celulitis eritematosa. Se cree que existen dos tipos de SSSS: una forma localizada, en la cual sólo existe un involucro «en parches» de la epidermis, y otra forma generalizada, en la que están involucradas áreas significativas, alejadas del sitio original de la infección. ${ }^{1-3}$

Se han documentado dos toxinas exfoliativas: A y $\mathrm{B}$, las cuales probablemente actúan como proteasas de la desmogleína-1 (DG-1), una importante proteína de unión entre queratinocitos que se encuentra solamente en la epidermis superficial. ${ }^{4,5}$

La cantidad relativa de DG-1 en la piel cambia con la edad y en parte explica la mayor frecuencia del síndrome de la piel escaldada por estafilococos en pacientes menores de cinco años. En la infancia es cuando el cuerpo adquiere inmunidad de por vida en forma de anticuerpos contra las exotoxinas de la cepa estafilocócica, por lo cual los menores de cinco años de edad (particularmente aquellos recién nacidos) tienen un alto riesgo de sufrir SSSS, reduciendo la posibilidad de presentarse en niños mayores y adultos. Por otra parte, los infantes son más susceptibles debido a la inmadurez del sistema inmune, y la función renal inmadura por eliminación deficiente de esas exotoxinas circulantes. . $^{1,2,6,7}$

La inmunidad reducida contra exotoxinas bacterianas y la depuración renal imperfecta aumentan la posibilidad de SSSS en los neonatos, ya que tales exotoxinas se eliminan a través de sus riñones. Esto se traduce en que, independiente de la edad y el sexo, las personas inmunocomprometidas y aquellos pacientes con insuficiencia renal están en riesgo de presentar SSSS. $1,2,7$

Los estudios preliminares sugerían que los fagos líticos del S. aureus subtipos 3A, 3B, 3C, 55 y 71 eran los únicos responsables de la producción de toxinas exfoliativas, pero ahora se sabe que todos los grupos de fagos son capaces de producirlas. ${ }^{7,8}$

Es importante realizar cultivos de los sitios dañados, así como de la nariz, la garganta y otros sitios potenciales del foco original del contagio para documentar la colonización por esa bacteria. En el caso de este texto, el exudado faríngeo y hemocultivo se reportaron negativos, consistente con el diagnóstico.

Se deben considerar los índices en aumento de inoculación por $S$. aureus resistente a meticilina adquirida en la comunidad (CA-MRSA), ya que la atención inmediata con antibióticos parenterales anti-estafilocócicos es esencial. Inicialmente desconociendo la susceptibilidad del microorganismo, dado que la mayoría de las infecciones estafilocócicas implicadas en el SSSS tienen penicilinasas y son refractarias a la penicilina, se debe iniciar alguna penicilina sintética resistente a la penicilinasa (como nafcilina, dicloxacilina). Si el organismo muestra susceptibilidad a la penicilina $\mathrm{G}$, debe usarse como tratamiento de elección. Alguna cefalosporina de primera generación puede usarse como alternativa. En pacientes alérgicos a la penicilina, los macrólidos o los aminoglucósidos pueden ser un sustituto. Las crecientes tasas de infecciones de $S$. aureus (CA-MRSA) resistentes a la meticilina, adquiridas en la comunidad, que al principio parecen ser tóxicas o que no responden a la nafcilina, $, 1,2,7,9,10$ requieren consideración para la terapia con vancomicina o linezolid.

En este caso, al principio se sospechó que se tratara de un $S$. aureus meticilino resistente debido a que recibió medicación con amoxicilina con clavulanato, pero teniendo los reportes de esos dos cultivos (lesiones perinasales y el hisopado de narinas, axilas, ingles), donde se aisló esa bacteria, es probable que los factores mencionados por la edad (inmadurez del sistema inmune, función renal inmadura condicionando eliminación deficiente de las exotoxinas circulantes) hayan sido responsables de la evolución tórpida que presentó.

El diagnóstico diferencial de SSSS puede imitar otras enfermedades exfoliantes como necrólisis epidérmica tóxica (TEN) y síndrome de Stevens Johnson (SJS). Se puede hacer la diferenciación evaluando la extensión de afección de las mucosas. Además, la ausencia de inflamación dérmica indica SSSS ya que TEN y SJS causan exfoliación de espesor completo. El dictamen se hace a menudo por exclusión si hay presencia de desgaste en ausencia de signos de infiltración. ${ }^{11-13}$

El pronóstico del síndrome de la piel escaldada estafilocócica en niños es excelente, dependiendo del estado inmunitario del huésped, la velocidad para iniciar el tratamiento adecuado, el curso de la infección y la aparición de complicaciones, con una curación completa que suele ocurrir en 10 a 14 días sin cicatrización significativa.

\section{CONCLUSIÓN}

Se expone el caso de un niño de tres años 11 meses con síndrome de piel escaldada, quien presentó adecuada respuesta al tratamiento una vez establecido, debido al manejo oportuno que recibió. Se da a conocer este reporte de caso por la importancia de tener 
An Med ABC. 2021; 66 (2): 146-150

un dictamen temprano de SSSS, teniendo en cuenta los diagnósticos diferenciales e iniciar el tratamiento correcto con antibióticos, así como mantener un adecuado estado hídrico para asegurar una rápida y completa recuperación.

\section{REFERENCIAS}

1. Cherry J, Demmler-Harrison GJ, Kaplan SL, Steinbach WJ, Hotez PJ. Feigin and Cherry's Textbook of pediatric infectious diseases. 8th edition. Philadelphia, PA: Saunders/Elsevier; 2018.

2. Long S. Principles and practice of pediatric infectious diseases. 5th edition. Philadelphia, PA: Elsevier; 2018.

3. Hubiche T, Bes M, Roudiere L, Langlaude F, Etienne J, Del Giudice P. Mild staphylococcal scalded skin syndrome: an underdiagnosed clinical disorder. Br J Dermatol. 2012; 166 (1): 213-215.

4. Hanakawa Y, Stanley JR. Mechanisms of blister formation by staphylococcal toxins. J Biochem. 2004; 136 (6): 747-750.

5. Ladhani S. Understanding the mechanism of action of the exfoliative toxins of Staphylococcus aureus. FEMS Immunol Med Microbiol. 2003; 39 (2): 181-189.

6. Ladhani S, Joannou CL, Lochrie DP, Evans RW, Poston SM. Clinical, microbial, and biochemical aspects of the exfoliative toxins causing staphylococcal scalded-skin syndrome. Clin Microbiol Rev. 1999; 12 (2): 224-242.

7. Mishra AK, Yadav P, Mishra A. A systemic review on staphylococcal scalded skin syndrome (SSSS): a rare and critical disease of neonates. Open Microbiol J. 2016; 10: 150159.

8. Deghorain M, Van Melderen L. The Staphylococci phages family: an overview. Viruses. 2012; 4 (12): 3316-3335.

9. Kaplan SL, Deville JG, Yogev R, Morfin MR, Wu E, Adler S et al. Linezolid versus vancomycin for treatment of resistant Gram-positive infections in children. Pediatr Infect Dis J. 2003; 22 (8): 677-686.

10. American Academy of Pediatrics. Staphylococcal infections. In: Kimberlin DW, Brady MT, Jackson MA, Long SS, eds. Red book: 2015 report of the Committee on Infectious Diseases. 30th ed. Elk Grove Village, IL: American Academy of Pediatrics; 2015. p. 715.

11. Aydin D, Alsbjorn B. Severe case of staphylococcal scalded skin syndrome in a 5-year-old child - case report. Clin Case Rep. 2016; 4 (4): 416-419.

12. Haasnoot PJ, De Vries A. Staphylococcal scalded skin syndrome in a 4-year-old child: a case report. J Med Case Rep. 2018; 12 (1): 20.

13. Chi CY, Wang SM, Lin HC, Liu CC. A clinical and microbiological comparison of Staphylococcus aureus toxic shock and scalded skin syndromes in children. Clin Infect Dis. 2006; 42 (2): 181-185. 